\title{
Strange Bedfellows: \\ What Really Defines Coalitions in International Climate Change Negotiations?
}

\author{
By Bryndís Arndal Woods* \\ Daði Már Kristófersson ${ }^{\dagger}$
}

\begin{abstract}
The purpose of this paper is to enhance our understanding of international climate change negotiations by providing insights into the structure and internal coherence of negotiation groups at COP 17 in Durban, with special reference to the relative importance of various negotiation issues. Discourse analysis is used to code the opening statements of participating countries in order to identify which negotiation issues are stressed. Statistical tools such as principal component analysis and cluster analysis are then used to identify the most important issues as well as to identify which negotiation groups emerge 'naturally' from the data. The coalitions uncovered in the analysis differ from existing negotiations groups and are more robust according to various measures. Existing coalitions are not homogenous (which has been demonstrated in the literature, e.g. Roberts, 2011; Vihma et al., 2011), nor are countries homogenous by region. This paper adds to our understanding by presenting a method of identifying groups and determining their internal stability. Our results demonstrate that the primary characteristic distinguishing coalitions was the degree that they emphasize the issues under negotiation.
\end{abstract}

\section{Introduction}

In 1992, the first international political response to the danger of climate change was made with the adoption of the United Nations Framework Convention on Climate Change (UNFCCC) which aimed to stabilize greenhouse gas concentrations in the atmosphere. The outcome of this meeting was the Kyoto Protocol, which legally required Annex I (developed) nations to reduce their emissions by $5.2 \%$ by 2012 from 1990 baseline levels (Roberts 2011). Since Kyoto, progress in the negotiations has stagnated. Small victories have been won, such as the Bali Action Plan at COP 13 in Indonesia in 2007 and the establishment of the Green Climate Fund at COP 16 in Mexico in 2010. However, negotiations have largely been characterized by failure and it is widely acknowledged that climate negotiations have contributed very little -

*PhD Researcher, University of Iceland/Aarhus University, Denmark.

${ }^{\dagger}$ University of Iceland, Iceland. 
if at all - to meaningful global carbon emission reductions (Ryding, 2012). This has caused a great deal of pessimism regarding the ability of the UNFCCC process to deliver results (Roberts, 2011; Tierney, 2012).

In the game theoretic literature, the failure to reach a binding international agreement since Kyoto is attributed to the non-cooperative nature of international negotiations. Forming coalitions is one type of strategy in international negotiations (Carraro, 1993). Economic theory operates on the assumption that countries join coalitions which they expect will provide them with the highest expected payoff from a specific negotiation outcome. A country is therefore more likely to join a coalition with common interests close to the country's preference. In general, more homogenous coalitions are likely to be more robust (Costantini et al., 2007).

Any attempt to quantify country preferences within the context of highly complex negotiations involves a degree of subjectivity and ideally, the 'true' preferences of each country would serve as the basis for clustering them together in groups, but we can only observe 'revealed preferences' in terms of formal negotiation positions, e.g. country's opening statements. A previous paper by the authors contains a soft analysis of the Durban round. ${ }^{1}$ The results of that study point to a gap worth filling: how to derive hard fact from soft data when applied to a highly complex issue where the need for simplification to understand the underlying problem is central. This paper addresses that question.

In the last fifteen years or so, a trend has emerged in the environmental economics literature which analyzes international negotiations whereby scholars use modified economic approaches to better account for 'reality' as such (e.g. Ward, Grundig and Zorick, 2001). This article builds upon this trend by applying a mixed-methodological approach to analyze a recent round of climate negotiations (COP 17) in Durban in 2011 to identify coalitions consisting of countries with overlapping preferences, based on formal negotiation positions. The results will contribute to our understanding of climate negotiations by providing insight into the structure and internal coherence of negotiation groups at COP 17, with special reference to the relative importance of various negotiation issues. A similar methodology was applied by Constantini et al. (2007) to analyze strategies and coalitions in WTO negotiations but this paper provides the first application on climate negotiations in the literature. ${ }^{2}$ Our methods allow the focus to remain on uncovering important political realities that enhance our understanding of complex negotiations.

The structure of this paper is as follows: Section 2 offers a brief background of the various methods employed in this paper. Section 3 describes the methodological approach of the study and Section 4 presents the results. In

\footnotetext{
${ }^{1}$ Access the article - "Towards a Better Understanding of Climate Change Negotiations" - at: http://www.irpa.is/article/view/1190.

${ }^{2}$ A similar methodological approach has been used to study WTO Agricultural negotiations (see Constantini, Crescenzi, Filippis and Salvatici, 2007) though this will be the first article to apply the approach to international climate change negotiations specifically.
} 
Section 5 we discuss the results, address relevant further applications and limitations of the study, and present concluding remarks.

\section{Background}

Game theory is a popular way to analyze climate negotiations, modeling the negotiations as a collection of players who have various demands, strategies, preferences, utility functions, and payoffs in an attempt to form stable coalitions which could effectively reduce global emissions (e.g. Tol and Osmani, 2009; Bréchet et al., 2010). There are two opposing views as to the nature of the game; one is based on cooperative game theory and the other is based on non-cooperative game theory. Today, most scholars agree that when dealing with international negotiations, the non-cooperative approach is better suited for the task because - as a rule - nations do not cooperate (Eyckmans and Finus 2003). In recent years, the form and application of game models have expanded rapidly, reflecting a trend whereby scholars have begun to critique and modify the 'traditional' approach in order to better account for important political and behavioral dynamics. ${ }^{1}$ However, even modified game theoretic analyses often split the world into politically irrelevant groupings, such as North and South (e.g. Caparrós, Péreau and Tazdait, 2004).

The participation of a great variety of agents negotiating over a great variety of issues for many years indicates that both discourse and cluster analyses are useful methodological approaches to adopt in the context of climate negotiations; both methodological approaches enable one to simplify complex relationships and identify trends within a large, complex data set. Scholars seeking to move beyond the economic and political insights of earlier work have examined participation in multilateral agreements and the 'accuracy' of current negotiation coalitions from a novel perspective (e.g. Egger, Jessberger and Larch, 2013). Such applications demonstrate the utility of these methodologies to better understand coalition formation according to pre-determined lines of inquiry - a body of work to which this paper contributes.

\section{Methods}

The data used in the discourse analysis were 137 country opening statements taken from the UNFCCC website (http://unfccc.int/). Discourse analysis has traditionally been associated with linguistics, but it has taken on a social orientation since the 1980s (Antaki, 2008). There are a full range of methods available, none of which claim general veracity. Applications of the

\footnotetext{
${ }^{1}$ Game designs which have been used to study climate negotiations include (but are not limited to): simple matrix games (e.g. Soroos, 1994), continuous games (e.g. Maler, 1990; Hoel, 1991), repeated games (e.g. Barrett, 1990; Ward, 1996) and sequential/dynamic games (e.g. Císcar and Soria, 2002; Forgó, Fulöp and Prill, 2005).
} 
methodology to climate change issues often focus on analyzing interpretations of discourse using mass media as raw data. This article will be the first to apply the methodology to climate negotiations discourse specifically and will also be the first to use those results as the basis for the subsequent cluster analysis.

The official negotiation positions of players may be influenced by tactical behavior whereby players conceal their true preference in order to prevent an undesirable outcome or gain political leverage. Aware of the limitations of the approach, we chose to treat countries' opening statements as a proxy for their true preferences. While tactical behavior is no doubt present, opening statements are usually made before the negotiation round begins and players have had the chance to see how negotiations are progressing. Each opening statement was coded for references to negotiation issues according to the same rubric. ${ }^{1}$ A new dataset was thereby created which included: the country or negotiation group making the statement, the number of times any issue was mentioned total, and the number of issues mentioned total.

Though the methods differ, the aims of this analysis are similar to those of Costantini et al. (2007): namely, using cluster analysis to compare 'natural' versus 'actual' coalitions and determining the degree of internal coherence within and across 'natural' and 'actual' negotiation groups. Cluster analysis does not require that the number of groups be known, nor does it require rules to assign membership to future observations. Both principal component analysis and cluster analysis can be applied to identify similarity among observations. Cluster analysis in particular has been applied in a wide array of contexts to identify latent structures (see Cohen et al., 2010; Wilks, 2011). Central to cluster analysis is the definition of distance and because a wide variety of distance functions exist, it is therefore an iterative process of identifying structure and not an automated task.

The discourse database was analyzed statistically, first by applying principal component analysis to identify latent structures and second, by allying cluster analysis to identify similar issues and to group countries. ${ }^{2}$ The analysis was conducted first on the issue dimension, to identify the key issues that separate countries and see if it was possible to simplify the issue space. It was then applied to the country dimension to identify groups.

Figure 1 shows the results of the cluster analysis of the issue space. The aim of this analysis is to identify the issues that contribute most to the variability in emphasis among countries. A successful clustering of issues should results in clusters with low internal variability and high variability between clusters. For that purpose, clusters are identified using semi-partial Rsquared, a direct measure of the homogeneity of merged clusters. If this measure of distance is small, it implies that the clusters being merged are homogenous. The results of the cluster analysis in Figure 1 demonstrate that

\footnotetext{
${ }^{1}$ Please contact the authors for any further information or additional materials regarding the methods employed.

${ }^{2}$ The results from the principal component analysis were only used to better understand the structure of the data. This structure was confirmed in the clustering and the results from the principal component analysis are therefore not reported.
} 
relatively few issues explain most of the variability in country emphasis; an important result in its own right.

Figure 1. The Results of the Clustering Procedure as applied to the Issue Dimension (across all countries), using Semi-structured R-squared to Measure Distance

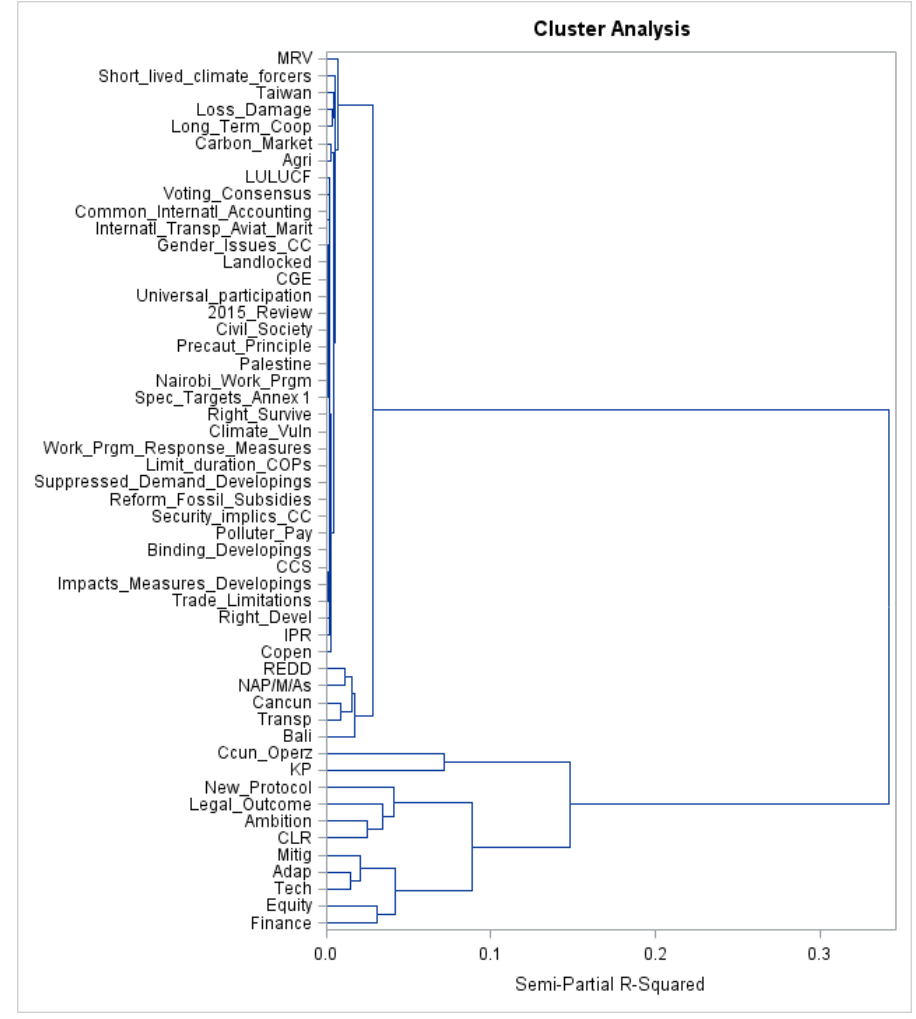

These results demonstrated that a large proportion of the negotiation issues do not help to distinguish countries from one another. A short list of central issues was generated based on these results. The country clustering was then run again using one long and two short lists of issues, to verify that omitted issues were truly irrelevant to the country classification. The results for country classification turned out to be very stable, regardless of the number of issues included. We utilized the shortest issue list for the remainder of the analysis in order to facilitate understanding by presenting the clearest possible picture. ${ }^{1}$

The purpose of the cluster analysis of countries is to identify which countries have similar emphasis on key issues in the climate negotiations. The most important property of the country clusters is therefore internal homogeneity. Again, the clusters were identified using semi-partial R-squared. Results are reported in Table 1.

Comparison of the results from the cluster analysis to existing definitions of groups in the climate negotiations are performed using several measures. Firstly, the characterizing emphasis on each issue in a group is measured using

${ }^{1}$ Shortest list: Finance, KP, Equity, CLR, Cancun Operationalization, New Protocol, Technology, Legal Outcome, Ambition. 
the average emphasis as a measure of homogeneity. Secondly, homogeneity is measured by estimating the average Euclidian distance between countries within clusters. Thirdly, different groupings are compared by estimating the percentage of explained variation in emphasis on different issues (R-squared) as well as share of explained distance. Finally, the quality of the groupings in explaining emphasis on issues is verified using the percentage of correct classifications in discriminant analysis.

The existing negotiation groups included in the analysis (and their associated acronyms) are as follows: Group of 77 and China (G77), Alliance of Small Island States (AOSIS), Least Developed Countries (LDCs), European Union (EU), The Umbrella Group (Umbrella), The BASIC Countries (BASIC), Environmental Integrity Group (EIG), Organization of the Petroleum Exporting Countries (OPEC), Countries of Central Asia and the Caucasus, Albania and Moldova (CACAM), Coalition for Rainforest Nations (CRN), the Bavarian Alliance for Latin America and the Caribbean (ALBA), the Like Minded Developing Countries (LMDC), the Association of Independent Latin American and Caribbean States (AILAC) and the Climate Vulnerable Forum $(\mathrm{CVF}) .^{1}$ It is important to acknowledge that this list serves as a snapshot in time, and that negotiation groups continue to emerge, evolve, and dissolve. We have attempted to capture those groups that played an important role at COP 17 in Durban.

\section{Results}

The cluster analysis produced seven 'natural' coalitions total (Table 1). What is immediately apparent is that clusters are not consistent by region with the exception of cluster 5, comprised of solely European nations.

\footnotetext{
${ }^{1}$ Note that the countries included in the analysis are not necessarily inclusive of group membership. Also note that there is a high degree of membership overlap between some of the groups which resulted in the necessity to create different classifications in order to generate the relevant statistics. For information regarding the members of the negotiation groups which were included in the analysis - due to group overlap and missing opening statements - please contact the authors.

Classification 1: G77, EU, Umbrella

Classification 2: AOSIS, EU, Umbrella, BASIC, EIG, OPEC, CACAM

Classification 3: LDCs, EU, Umbrella, EIG, CACAM, ALBA, LMDC, AILAC

Classification 4: CRN, EU, Umbrella, BASIC, EIG, CACAM

Classification 5: CVF, EU, Umbrella, BASIC, EIG, OPEC, CACAM, ALBA
} 
Table 1. 'Natural Coalitions' from Cluster Analysis*

\begin{tabular}{|c|c|}
\hline Cluster 1 & $\begin{array}{l}\text { Afghanistan, Angola, Belize, Botswana, Burundi, China, Comoros, } \\
\text { Cuba, Guinea, Honduras, Indonesia, Iran, Lesotho, Mali, Mauritania, } \\
\text { Mauritius, Moldova, Montenegro, Paraguay, Russia, Saudi Arabia, } \\
\text { Sierra Leone, Somalia, Spain, Sri Lanka, St. Kitts and Nevis, Thailand, } \\
\text { Togo, Ukraine, Zimbabwe }\end{array}$ \\
\hline Clu & $\begin{array}{l}\text { Bolivia, Burkina Faso, Canada, Costa Rica, Democratic Republic of the } \\
\text { Congo, Ecuador, El Savador, Eritrea, Gabon, Georgia, Greece, India, } \\
\text { Ireland, Israel, Kazakhstan, Lao, Lebanon, Liechtenstein, Mongolia, } \\
\text { New Zealand, Nicaragua, Niue, Panama, Papua New Guinea, Peru, } \\
\text { Slovakia, Turkey, Tuvalu, Uruguay, Venezuela, Vietnam }\end{array}$ \\
\hline & $\begin{array}{l}\text { Algeria, Chad, Denmark, Ivory Coc } \\
\text { Netherlands, Rwanda, Tanzania, Tuı }\end{array}$ \\
\hline & $\begin{array}{l}\text { Australia, Austria, Barbados, Belgium, Bhutan, Czech Republic, } \\
\text { Dominica, Estonia, Fiji, Finland, Germany, Iceland, Jamaica, Japan, } \\
\text { Kiribati, Maldives, Marshall Islands, Mexico, Micronesia, Monaco, } \\
\text { Nigeria, Norway, Palau, Philippines, Samo, Serbia, Seychelles, } \\
\text { Slovenia, Solomon Islands, Swaziland, Sweden, Tonga }\end{array}$ \\
\hline & $\begin{array}{l}\text { Belarus, Cyprus, France, Italy, Luxembourg, Malta, Portugal, Romania, } \\
\text { United Kingdom }\end{array}$ \\
\hline & Brazil, Ethiopia, Senegal \\
\hline Cluster 7 & $\begin{array}{l}\text { Bangladesh, Benin, Central African Republic, Colombia, Ghana, } \\
\text { Kenya, Liberia, Malawi, Namibia, Nepal, Pakistan, Republic of the } \\
\text { Congo, Singapore, South Africa, Suriname, Timor-Leste, Trinidad and } \\
\text { Tobago, Uganda, Zambia }\end{array}$ \\
\hline
\end{tabular}

*Players in italics are core cluster members; meaning present in all 3 versions of the analysis conducted (full list of issues, short list of issues, and shortest list of issues)

What distinguishes groups from one another is the emphasis (or lack of emphasis) on various negotiation issues. Table 2 presents the cohesiveness as well as the issues stressed by each cluster. Clusters 2 (a mix of countries from every continent) and 4 (Japan, European countries, and a mix of developing countries) both stress no issues, though cluster 4 emphasizes issues slightly more strongly overall. Cluster 3 (the U.S. plus a mix of African and European countries) stresses one issue: Cancun operationalization. Clusters 1 and 5 both stress two issues. Cluster 1 (China, Middle Eastern nations, and developing African nations) stresses the Kyoto Protocol and finance. Cluster 5 (EU countries) stresses a new Protocol and a legal outcome. Cluster 6 (Brazil, Ethiopia and Senegal) stresses three issues: the Kyoto Protocol, Cancun operationalization, and finance. Finally, cluster 7 (African and Asian developing countries as well as South Africa) emphasizes four issues: Cancun operationalization, finance, equity and the Kyoto Protocol. 
Table 2. Number of Countries in 'Natural' Coalitions, Cohesiveness of 'Natural' Coalitions as measured by the Average Distance from Cluster Centroid, and Average Number of Issue-mentions among the 'Natural' Coalitions where issues stressed $(X \geq 1.5)$ by each Cluster in Bold*

\begin{tabular}{|c|c|c|c|c|c|c|c|c|c|c|c|}
\hline & \multirow[b]{2}{*}{ 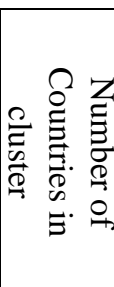 } & \multirow[b]{2}{*}{ 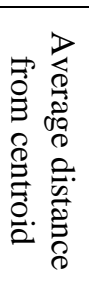 } & \multicolumn{9}{|c|}{ Average number of mentions of issues by cluster members } \\
\hline & & & 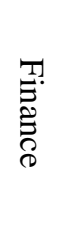 & $\begin{array}{ll} & 0 \\
0 & \pi \\
0 & 0 \\
0 & 0 \\
0 & 0 \\
0 & 0\end{array}$ & $\begin{array}{l}\text { T) } \\
\text { E. }\end{array}$ & 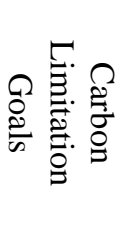 & 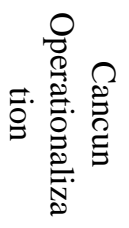 & $\begin{array}{l}Z \\
0 \\
0 \\
z \\
0 \\
0 \\
0 \\
0 \\
0 \\
0\end{array}$ & 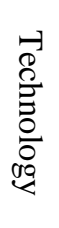 & 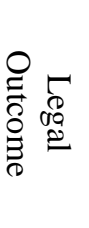 & : \\
\hline Cluster 1 & 30 & 2.2 & 1.6 & 2.2 & 1.1 & 0.5 & 1.4 & 0.3 & 1.2 & 0.4 & 0.4 \\
\hline Cluster 2 & 31 & 1.6 & 0.6 & 0.7 & 0.5 & 0.2 & 0.4 & 0.2 & 0.3 & 0.5 & 0.1 \\
\hline Cluster 3 & 12 & 1.3 & 0.3 & 1.2 & 0.3 & 0.1 & 3.0 & 0.1 & 0.1 & 0.1 & 0.0 \\
\hline Cluster 4 & 33 & 2.2 & 0.4 & 1.2 & 0.2 & 1.0 & 1.2 & 0.9 & 0.1 & 1.2 & 0.8 \\
\hline Cluster 5 & 9 & 1.7 & 0.2 & 1.4 & 0.6 & 0.8 & 0.4 & 3.3 & 0.4 & 1.9 & 0.1 \\
\hline Cluster 6 & 3 & 2.2 & 1.7 & 4.7 & 1.3 & 0.3 & 4.0 & 0.7 & 0.7 & $1.7 *$ & 0.7 \\
\hline Cluster 7 & 19 & 2.5 & 2.3 & 1.7 & 2.3 & 0.5 & 3.9 & 0.3 & 1.1 & 1.4 & 0.2 \\
\hline
\end{tabular}

*The associated variance on issue dimensions marked with an asterisk were too high to present the result as meaningful.

Table 3 presents the issue averages of the negotiation groups active within the UNFCCC. There is a great deal more overlap across issue emphasis than among the 'natural' coalitions which makes it more difficult to identify what sets groups apart from one another. The exception is the EU which emphasizes the same two issues - a new Protocol and a legal outcome - as 'natural' cluster 5, comprised of exclusively EU nations. Otherwise, seven of the nine issues are emphasized by only two coalitions or fewer. The remaining two issues - the Kyoto Protocol and Cancun operationalization - are emphasized by ten of the fourteen coalitions.

In order to determine the cohesiveness of our clusters versus existing negotiation-groups, we determined the 'centroid' of each cluster and calculated the average distance of member countries from the group's centroid (Table 2 displays the results for the natural coalitions and Table 3 displays the results for the existing coalitions). The 'natural' clusters revealed in our analysis display relatively high internal cohesion. When one compares the 'natural' clusters to existing negotiation-groups, the distance from the group's centroid are higher among existing coalitions overall, though not across the board. This trend is more robust when one considers the relative size of the coalitions. 
Table 3. Number of Countries in Existing Coalitions, Cohesiveness of Existing Coalitions as measured by the Average Distance from Cluster Centroid, and Average Number of Issue-mentions among the Existing Coalitions where Issues stressed $(X \geq 1.5)$ by each Cluster in Bold*

\begin{tabular}{|c|c|c|c|c|c|c|c|c|c|c|c|}
\hline & \multirow[b]{2}{*}{ 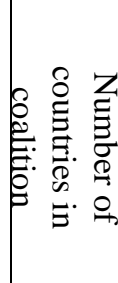 } & \multirow[b]{2}{*}{ 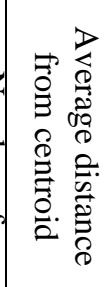 } & \multicolumn{9}{|c|}{ Average number of mentions of issues by cluster members } \\
\hline & & & 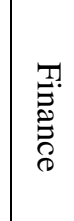 & 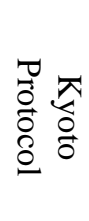 & $\begin{array}{l}\frac{\pi}{2} \\
\stackrel{0}{E} .\end{array}$ & 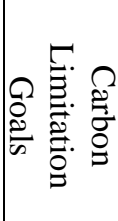 & 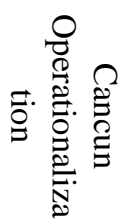 & $\begin{array}{l}Z \\
0 \\
0 \\
z \\
0 \\
0 \\
0 \\
0 \\
0 \\
0\end{array}$ & 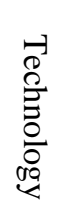 & 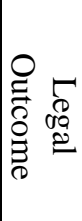 & : \\
\hline G77 & 90 & 2.7 & 1.3 & 1.7 & 1.0 & 0.5 & 1.9 & 0.2 & 0.7 & 0.8 & 0.4 \\
\hline AOSIS & 24 & 2.6 & 1.0 & 1.3 & 0.5 & 1.1 & 1.5 & 0.4 & 0.3 & 0.9 & 0.9 \\
\hline LDCs & 25 & 2.6 & 1.4 & 2.1 & 1.2 & 0.4 & 2.1 & 0.0 & 0.8 & 0.9 & 0.1 \\
\hline EU & 22 & 2.3 & 0.2 & 1.1 & 0.3 & 0.7 & 1.0 & 1.7 & 0.2 & 1.5 & 0.3 \\
\hline Umbrella & 9 & 2.2 & 0.3 & 1.6 & 0.2 & 0.6 & 1.0 & 1.3 & 0.3 & \begin{tabular}{|l|}
0.3 \\
\end{tabular} & 0.1 \\
\hline BASIC & 4 & 2.8 & 1.8 & 2.8 & 1.5 & 0.0 & $3.3^{*}$ & 0.3 & 1.3 & 1.0 & 0.3 \\
\hline EIG & 4 & 2.1 & 0.8 & 1.0 & 0.0 & 0.8 & 1.8 & 0.5 & 0.5 & 0.3 & 0.5 \\
\hline OPEC & $\begin{array}{c}7 \\
(6)\end{array}$ & $\begin{array}{c}2.3 \\
(2.4)\end{array}$ & 1.3 & 1.9 & 0.6 & 0.1 & 1.4 & 0.0 & 1.0 & 0.3 & 0.1 \\
\hline CACAM & 4 & 2.5 & 0.5 & 1.3 & 0.5 & 0.3 & 1.8 & 0.0 & 1.0 & 0.5 & 0 \\
\hline CRN & 33 & 2.6 & 1.5 & 1.4 & 1.2 & 0.5 & 2.1 & 0.3 & 0.7 & 0.8 & 0.3 \\
\hline ALBA & 7 & 2.0 & 1.0 & 1.4 & 0.4 & 0.4 & 0.7 & 0.0 & 0.6 & 0.3 & 0.1 \\
\hline LMDC & 14 & 2.9 & 1.4 & 1.8 & 1.5 & 0.4 & $2.2 *$ & 0.7 & 1.0 & 0.5 & 0.3 \\
\hline AILAC & 4 & 2.3 & 0.8 & 1.0 & 0.3 & 0.5 & $1.5^{*}$ & 0.3 & 0.8 & 1.0 & 0.3 \\
\hline $\mathrm{CVF}$ & 17 & 3.0 & 1.3 & 1.2 & 0.9 & 0.6 & 2.0 & 0.5 & 0.6 & 0.9 & 0.5 \\
\hline
\end{tabular}

*The associated variance on issue dimensions marked with an asterisk were too high to present the result as meaningful. OPEC was included in classifications 2 and 5; results from classification 5 are in parentheses.

Next, we conducted a discriminate analysis which creates a linear aggregation of the issues and generates an index for each group classification which can be used to predict which players fall into which group based on their position on those issues. The results demonstrate that the 'natural' coalition classification is much stronger in this regard than all other classifications of existing negotiation groups (Table 4). It is important to emphasize the dependence of this measure on the number of clusters included in the classification. For instance, it would not be an impressive result if group membership were predicted $100 \%$ correctly if there were only one group. However, the fact that the 'natural' coalition classification outperforms all the others with a total of 7 clusters and 137 countries - more than any other classification - enables one to put more faith in the result.

Table 4 also demonstrates that the 'natural' clusters explain a significantly higher percentage of the variance across issues. The 'natural' coalition classification explains a larger percentage of variance than existing coalitions across all issues (taken individually), with the exception of classification 3 
within the finance issue dimension. Approximately $50 \%$ of the distance over all issues (taken together) is explained by the 'natural' clusters, whereas the existing negotiation groups' classifications explain only $12 \%, 21 \%$ or $22 \%$; an indication that our classification is a better fit when countries are clustered according to their negotiation position (Table 4).

Table 4. The number of countries and clusters included in all classification systems; discriminant function and the percent of countries correctly classified into 'natural' clusters and classifications $1-5$; $R$-squared values by issue of our classification of 'natural' clusters, the five classifications of existing clusters; and the total $R$-squared value which is the explained distance over all issues for each classification system*

\begin{tabular}{|c|c|c|c|c|c|c|c|c|c|c|c|c|c|}
\hline & \multirow[b]{2}{*}{ 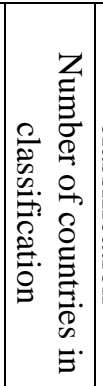 } & \multirow[b]{2}{*}{ 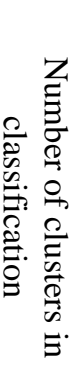 } & \multirow[b]{2}{*}{ 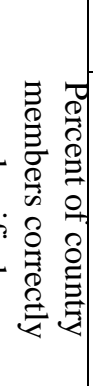 } & \multicolumn{10}{|c|}{$\mathrm{R}^{2}$ for explained variation in emphasis on issues } \\
\hline & & & & 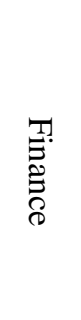 & $\begin{array}{l}\text { त् } \\
0 \\
0 \\
0 \\
0 \\
0 \\
0 \\
0 \\
0\end{array}$ & 焉 & 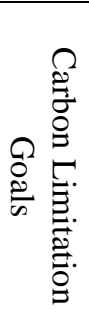 & 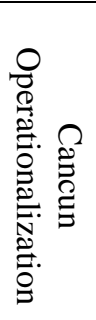 & 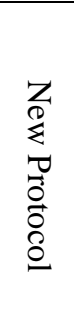 & 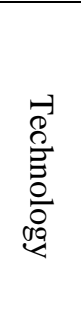 & 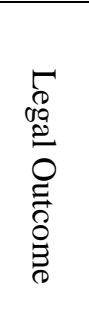 & : & 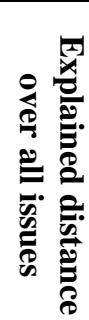 \\
\hline $\begin{array}{c}\text { 'Natural' } \\
\text { Coalitions }\end{array}$ & 137 & 7 & $93 \%$ & $53 \%$ & $43 \%$ & $44 \%$ & $19 \%$ & $70 \%$ & $58 \%$ & $38 \%$ & $35 \%$ & $20 \%$ & $49 \%$ \\
\hline $\begin{array}{c}\text { Classification } \\
1\end{array}$ & 119 & 3 & $89 \%$ & $19 \%$ & $4 \%$ & $9 \%$ & $1 \%$ & $7 \%$ & $40 \%$ & $7 \%$ & $15 \%$ & $2 \%$ & $12 \%$ \\
\hline $\begin{array}{c}\text { Classification } \\
2\end{array}$ & 74 & 7 & $64 \%$ & $25 \%$ & $13 \%$ & $19 \%$ & $16 \%$ & $15 \%$ & $37 \%$ & $23 \%$ & $29 \%$ & $17 \%$ & $21 \%$ \\
\hline $\begin{array}{c}\text { Classification } \\
3\end{array}$ & 89 & 8 & $57 \%$ & $53 \%$ & $15 \%$ & $27 \%$ & $4 \%$ & $17 \%$ & $36 \%$ & $15 \%$ & $22 \%$ & $6 \%$ & $21 \%$ \\
\hline $\begin{array}{c}\text { Classification } \\
4\end{array}$ & 76 & 6 & $66 \%$ & $37 \%$ & $13 \%$ & $18 \%$ & $5 \%$ & $20 \%$ & $33 \%$ & $15 \%$ & $26 \%$ & $5 \%$ & $22 \%$ \\
\hline $\begin{array}{c}\text { Classification } \\
5\end{array}$ & 72 & 8 & $57 \%$ & $28 \%$ & $14 \%$ & $22 \%$ & $7 \%$ & $20 \%$ & $37 \%$ & $20 \%$ & $31 \%$ & $6 \%$ & $22 \%$ \\
\hline
\end{tabular}

*See Footnote 7 for an in-depth explanation of the classification systems. The total R-squared is a multi-dimensional statistic based on distance whereas the individual issue $\mathrm{R}$-squared statistics are single-dimensional and not based on distance.

\section{Discussion/Conclusions}

The fragmentation of formal UNFCCC negotiation groups has been the focus of much study in recent years. ${ }^{1}$ The results of this study confirm the heterogeneity within existing negotiation groups but took the analysis a step further by examining which coalitions emerge 'naturally' when we allow countries' negotiation positions to serve as the basis for cluster formation. This allowed us to quantify the manner in which existing groups are heterogeneous

${ }^{1}$ See, for example, Roberts, 2011; Betzold, Castro and Weiler, 2011; Vihma et al., 2011; Oberthur and Dupont, 2011. 
by providing insights into the structure and internal coherence of the 'natural' bargaining coalitions as compared to existing coalitions. Analyzing which groups emerge 'naturally' from the data and what distinguishes them from one another is made possible by the novel methodological approach employed; one would not obtain such results from qualitative nor quantitative methods alone.

Our first result - which was also part of the methodological approach was that relatively few negotiation issues explain most of the variability in country emphasis. 36 of the 52 issues included in the analysis did not help at all to distinguish countries from one another. Nine very stable 'natural' clusters emerged regardless of whether they were clustered according to all 52 issues, a short list of 14 issues, or the shortest list of 9 issues. The issues which contributed most to variability were generally those issues which were mentioned most often.

Our results confirm the problematic nature of using existing negotiation groups or clustering countries by region in analyses of climate negotiations as this may obscure the issues which are truly under consideration and entail assumptions regarding homogeneity of groups which are at odds with reality (e.g. Caparrós, Péreau and Tazdait, 2004 and Tol and Osmani, 2009). ${ }^{1}$ Our results also underline the problematic assumption that countries join the coalition that aligns most closely with their own position; this does not appear to be the norm. The explanation may be more complex than strategic behavior alone, but it is nevertheless an important dynamic to understand when conducting analyses of climate negotiations.

The existing clusters exhibit many of the same trends in issue emphasis to the natural clusters. First, the majority of clusters emphasize very few issues. All but one of the existing clusters and 4 of the 7 'natural' clusters emphasize two issues or fewer. Among both the existing and 'natural' clusters there are two clusters which emphasize no issues at all. ALBA and AILAC, relatively small coalitions with 7 and 4 countries respectively, emphasize nothing. Clusters 2 and 4 which are the two largest 'natural' clusters with 31 and 33 countries respectively (and are comprised of countries from every single continent), also emphasize nothing. 4 of the 14 existing coalitions emphasize only one issue, which in each case is either Cancun operationalization or the Kyoto Protocol. 1 of the 7 'natural clusters' - Cluster 3, which included the U.S. as well as progressive European nations like Denmark and developing African countries like Rwanda - emphasized only operationalizing decisions already made in Cancun. The EU stands out as the most easily distinguishable and exceptional cluster among both the existing and 'natural' clusters. In both cases the EU cluster emphasizes establishing a (1) new and (2) legally-binding Protocol and is the only cluster to emphasize either of those issues. It is the clearest and most meaningful overlap between our clusters and existing negotiation groups. One final overlapping trend is interesting to note: 3 of the 9 issues included in the analysis (and are also among the most mentioned issues

\footnotetext{
${ }^{1}$ For example, Bréchet et al., 2010; Eyckmans and Finus, 2003 and Carraro, Eyckmans and Finus, 2005 all divide the world into six regions: USA, Japan, European Union, China, Former Soviet Union, and Rest of the World.
} 
overall) - carbon limitation goals, technology and ambition - were not emphasized at all among either the existing or the 'natural' clusters.

At this point, the trends among the remainder of the 'natural' clusters diverge from the existing coalitions. These clusters do not find any counterparts among the existing clusters (with the exception of BASIC which emphasizes finance, the Kyoto Protocol and equity). Cluster 1 - comprised of big developing countries like China, Middle Eastern countries like Iran, and least developed countries like Botswana - emphasized finance and the Kyoto Protocol. The two remaining 'natural' clusters -6 and $7-$ had the strongest and second-strongest emphasis, respectively. Cluster 6 stressed the Kyoto Protocol, Cancun operationalization, and finance. It was also the smallest cluster by far, comprised of Brazil, Ethiopia, and Senegal. Cluster 7 emphasized the largest number of issues: Cancun operationalization, finance, equity and the Kyoto Protocol. This group was made up of a smattering of least developed countries like Zambia and Liberia as well as South Africa.

We have shown that existing negotiation coalitions are not the most robust when examined according to similarity across negotiation issues. The 'natural' clusters uncovered in our analysis displayed higher internal cohesion than existing coalitions, explained a much higher percentage of the variance across issues, and had their membership more consistently correctly classified using discriminant analysis. This indicates that analyzing the negotiation issues that countries emphasize is an important way to understand the inner workings of climate negotiations. Ultimately, the primary distinguishing characteristic between clusters was the degree to which they emphasized issues: those who do and those who don't. This result indicates that indifference and a desire to maintain the status quo is not only common, but that it is what holds some of our clusters together.

Our results demonstrate that grouping countries according to their formal negotiation position makes strange bedfellows. Not only are curious alliances struck between nations that otherwise have little in common, but there is also a curious lack of alliances between nations that one would have every reason to believe would be grouped together. The sweeping indifference countries displayed regarding progressive, global action on climate change may be a depressing result, but it is important to acknowledge such dynamics exist if we are to begin to rectify them. There is too much at stake for scholars to look the other way if countries' presumed to agree with one another on coherent environmental policies actually do not.

We realize that our procedures are somewhat subjective and that a change in the data used (e.g. a different classification system to code the opening statements, using closing statements, analyzing a different negotiation round, etc.) might yield different results. The opening statement format is not

\footnotetext{
${ }^{1}$ There may be reasons other than indifference which would lead a country to emphasize very few issues in its opening statement. For example, some countries emphasize the impacts of climate change on their own country in order to paint a picture. Because of the coding method used in this paper, such opening statements would appear to be "indifferent" though they are not.
} 
standardized, which means that our results may be unstable over time; for example, it may be the case that the issues which were most heavily stressed in this analysis are different than in another negotiation round. A meaningful extension of this study would be to apply either the same or a similar method to multiple negotiation rounds in order to determine whether the results are stable or unstable over time.

Our basic purpose is not to provide a "final" classification of countries but to focus on a higher-order comparison that combines qualitative and quantitative analytical methods in a straight-forward manner and is able to provide an enhanced understanding of the degree of overlap with regard to countries' negotiation positions and the internal coherence of coalitions. It is our hope that this study contributes to our overall understanding of coalition formation and the 'sustainability' of negotiation groups as they currently stand as well as contributing to the general methodological literature on analyzing soft data.

\section{References}

Antaki, C., 2008. Discourse Analysis and Conversation Analysis. In P. Alasuutari, L. Bickman, and J. Brannen, The SAGE Handbook of Social Research Methods. Sage Publications Ltd. pp. 431-447

Bréchet, T., Eyckmans, J., Gérard, F., Marbaix, P., Tulkens, H., and van Ypersele, J.P., 2010. The impact of the unilateral EU commitment on the stability of international climate agreements. Climate Policy, 10(2), pp. 148-166.

Caparrós, A., Péreau, J.-C., and Tazdait, T., 2004. North-South climate change negotiations: A sequential game with asymmetric information. Public Choice, 121(3/4), pp. 455-480.

Carraro, C., 1993. Strategies for the international protection of the environment. Journal of Public Economics, 52(3), pp. 309-328.

Cohen, M., Grossman, A., Morabito, D., Knudson, M., Butte, A. and Manley, G., 2010. Identification of complex metabolic states in critically injured patients using bioinformatic cluster analysis. Critical Care, 14(1), R10.

Costantini, V., Crescenzi, R., De Filippis, F. and Salvatici, L., 2007. Bargaining Coalitions in the WTO Agricultural Negotiations. The World Economy, 30(5), pp. 863-891.

Egger, P., Jessberger, C. and Larch, M. Impacts of Trade and the Environment on Clustered Multilateral Environmental Agreements. The World Economy, 36(3), pp. 331-348.

Eyckmans, J., and Finus, M., 2003. New Roads to International Environmental Agreements: The Case of Global Warming. Leuven: K.U. Leuven.

Roberts, J., 2011. Multipolarity and the new world (dis)order: US hegemonic decline and the fragmentation of the global climate regime. Global Environmental Change 21(3), pp. 776-784.

Ryding, T., 2012. Climate protection between hope and despair: 20 years of the UNFCCC. Greenpeace International. Available at: http://www.greenpeace.org/ international/ Global/international/publications/RioPlus20/20-Years-of-UNFCC C.pdf. [Accessed 18 May 2014]. 
Tierney, J., 2012. Climate Proposal Puts Practicality Ahead of Sacrifice. New York Times, [online] Available at: < http://www.nytimes.com/2012/01/17/science/coun tering-climate-change-without-waiting-for-apayoff.html? $r=1$ andpagewanted=all $>$ [Accessed on 18 May 2014].

Tol, R., Osmani, D., 2009. Toward Farsightedly Stable International Environmental Agreements. Environmental Economics and Management, 11(3), pp. 1-34.

Vihma, A., Mulugetta, Y., and Karlsson-Vinkhuyzen, S., 2011. Negotiating solidarity? The G77 through the prism of climate change negotiations. Global Change, Peace and Security, 23(3), pp. 315-334.

Ward, H., Grundig, F., and Zorick, E., 2001. Marching at the Pace of the Slowest: a Model of International Climate Change Negotiations. Political Studies, 49(3), pp. 438-461.

Wilks, D.S., 2011. Cluster Analysis. International Geophysics, vol.100, pp.603-616. 